\title{
Photoluminescence Properties of Zinc Oxide Film with Local Epitaxial Growth on Silicon Using Aluminum Nitride Buffer Layer
}

\begin{abstract}
LiU Li, LiU Sha AND YANG YUAN*
College of Petroleum and Chemical Engineering, BaYin GuoLeng Technology College, Xinjiang 841000, China

(Received November 19, 2019; revised version January 8, 2020; in final form January 28, 2020)

In this work, we report the preparation of zinc oxide $(\mathrm{ZnO})$ film with local epitaxial growth on silicon substrate at room temperature using aluminium nitride $(\mathrm{AlN})$ as buffer layer. $\mathrm{ZnO}$ and $\mathrm{AlN}$ films were deposited by $\mathrm{RF}$ and DC magnetron sputtering, respectively. The microstructures of $c$-axis oriented ZnO films deposited on (100) Si and AlN/Si substrates were studied by scanning electron microscopy, transmission electron microscopy, and X-ray diffraction. In addition, the effect of AlN buffer thicknesses (600 and $1400 \mathrm{~nm}$ ) on photoluminescence properties of $400 \mathrm{~nm}$ thick $\mathrm{ZnO}$ deposits were also studied. Indeed, the deposition of $\mathrm{ZnO}$ on $\mathrm{AlN}$ buffer leads to a red shift of the band gap emission of $\mathrm{ZnO}$ compared to $\mathrm{ZnO}$ deposited without buffers. Moreover, the defects emission intensities and nature were also affected. The evolution of $\mathrm{ZnO} \mathrm{PL}$ properties due to AlN buffer insertion is discussed with respect to the difference in the structural properties of $\mathrm{ZnO}$ film with and without AlN buffers.
\end{abstract}

DOI: 10.12693/APhysPolA.137.1110

PACS/topics: aluminium nitride, zinc oxide, buffer layer, photoluminescence, epitaxial growth

\section{Introduction}

Zinc oxide $(\mathrm{ZnO})$ is an attractive $\mathrm{II}-\mathrm{VI}$ semiconductor, with a direct band gap of about $3.3 \mathrm{eV}$ and high exciton binding energy $(60 \mathrm{meV})[1-3]$. Therefore, it is suitable for optoelectronic devices, including ultravioletblue semiconductor lasers, light emitting diodes, and solar cells [1-4]. $\mathrm{ZnO}$ thin films have been grown using several deposition techniques, such as pulsed laser deposition [5], molecular beam epitaxy [6], MOCVD [7] etc. Furthermore, epitaxial growth of $\mathrm{ZnO}$ on silicon substrate $(\mathrm{Si})$ is sometimes necessary in order to integrate these films in microelectronic devices. Nonetheless, due to the lattice mismatch between $\mathrm{ZnO}$ and $\mathrm{Si}$, it is hard to grow epitaxial $\mathrm{ZnO}$ directly on $\mathrm{Si}$ substrate, at temperature compatible with that used in semiconductor technology. One possible way to overcome this limitation is by using a buffer layer with small lattice mismatch compared to that of $\mathrm{ZnO}$. For instance, magnesium oxide $(\mathrm{MgO})$ [8], gallium nitride [9], and aluminium nitride (AlN) $[8,10,11]$, have been employed as buffer layers in order to obtain epitaxial growth of $\mathrm{ZnO}$ film. In particular AlN has a hexagonal wurtzite structure similar to that of zinc oxide, with lattice parameters of $a=0.311 \mathrm{~nm}$ and $c=0.498 \mathrm{~nm}[8]$. On the other hand, $\mathrm{ZnO}$ has also hexagonal wurtzite structure, with lattice parameters of $a=0.324 \mathrm{~nm}$ and $c=0.520 \mathrm{~nm}[8]$. Therefore, $\mathrm{ZnO}$ thin films can be grown on AlN by means of lattice-matching epitaxy, since the lattice misfit between these two materials is less than $4 \%$ [8]. Although, the epitaxial growth of $\mathrm{ZnO}$ has been reported in literature [8-11]. However, the

*corresponding author; e-mail: 66299704@qq.com optical properties of epitaxial $\mathrm{ZnO}$ are not well developed in literature. For example, very few studies have been reported on the photoluminescence (PL) performance of epitaxial ZnO. For example, Fujita et al. [12] have reported the PL emission intensity improvement of epitaxial $\mathrm{ZnO}$ deposited on $\mathrm{MgO}$ buffer layer. Xiong et al. [10] have also reported the PL improvement of grown $\mathrm{ZnO}$ on AlN buffer. In most of cases, the PL improvement of epitaxial $\mathrm{ZnO}$ is due to the structural quality improvement compared to $\mathrm{ZnO}$ deposited directly without epitaxial growth. However, epitaxial growth does not always lead to an enhancement of PL intensity, as it will be discussed in the present work.

In this work, we report on the epitaxial growth of $\mathrm{ZnO}$ thin films at low temperature $\left(<100^{\circ} \mathrm{C}\right)$ on silicon substrates using an AlN buffer layer. The $\mathrm{ZnO}(400 \mathrm{~nm}$ thick) thin films were deposited by RF-sputtering on two AlN buffers with two different thicknesses of $600 \mathrm{~nm}$ or $1400 \mathrm{~nm}$. We show that even for epitaxial $\mathrm{ZnO}$, we did not obtain a significant improvement of band gap PL emission. In addition, the defect emissions were modified after AlN buffer insertion. The difference of PL signal before and after AlN insertion is discussed with respect to the structural modification of the $\mathrm{ZnO}$ deposit with and without AlN buffer. Our results add a piece of information for the optical properties of epitaxial $\mathrm{ZnO}$ film with specific structural configuration.

\section{Experimental procedure}

\subsection{Deposition of AlN buffers}

AlN films were deposited at room temperature, on (100) oriented silicon substrates, by reactive DC-sputtering in a customized reactor equipped with 


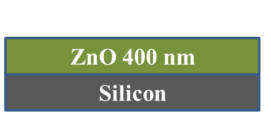

(a)

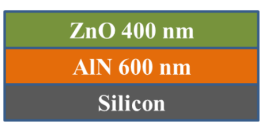

(b)

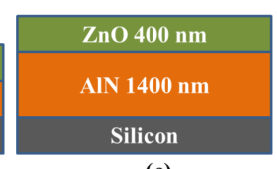

(c)
Fig. 1. Schematic design of the investigated samples. The $\mathrm{ZnO}$ films were grown on (a) silicon substrate, (b) $600 \mathrm{~nm}$ thickness AlN/silicon templates, (c) $1400 \mathrm{~nm}$ thickness AlN/silicon templates using RF-sputtering.

a 2 inch magnetron cathode, with a pure aluminium target at deposition pressure of 2 mTorr. Prior to deposition, the substrates were ultrasonically cleaned in acetone and ethanol, then dried under nitrogen gas flux. The sputtering target (aluminium) was $99.99 \%$ in purity with 2 " in diameter, held on a water-cooled magnetron cathode. The distance between the target and substrate holder was $3 \mathrm{~cm}$. The system was pumped to a base pressure of $4.5 \times 10^{-5}$ Torr with a turbo-molecular pump before introducing argon (99.99\%) and nitrogen (99.99\%) gases. The total gas flux rate during deposition was maintained constant at $40 \mathrm{sccm}$ while that of the reactive $\mathrm{N}_{2}$ gas was fixed at $35 \%$. The substrates were at floating potential and were not additionally heated nor cooled during the deposition. Deposition times of $20 \mathrm{~min}$ and $50 \mathrm{~min}$ were chosen, in order to obtain AlN buffer with two different thicknesses of $600 \mathrm{~nm}$ and $1400 \mathrm{~nm}$, respectively.

\subsection{Deposition of $\mathrm{ZnO}$}

Zinc oxide ( $\mathrm{ZnO})$ films were deposited on (100) silicon substrate and AlN (600 and $1400 \mathrm{~nm}$ thick) buffer layers, by RF-sputtering from 3" diameter of $\mathrm{ZnO}$ target. The target-sample distance was fixed at $2.6 \mathrm{~cm}$. Argon $(99.99 \%)$ as an inert gas was introduced through a mass flow controller. Sputtering deposition was performed under a gas pressure of 2.5 mTorr without intentional heating. The input power was kept at $200 \mathrm{~W}$. The $\mathrm{ZnO}$ thickness $(400 \mathrm{~nm})$ was measured with a surface profilometer on silicon substrate loaded in the same reactor during $\mathrm{ZnO}$ deposition. The schematic structures of the samples are shown in Fig. 1. The samples are assigned as (a), (b) and (c) for $\mathrm{ZnO}$ deposited on Si without AlN buffer and for $\mathrm{ZnO}$ deposited on $600 \mathrm{~nm}$ and $1400 \mathrm{~nm}$ thick AlN buffers, respectively.

\subsection{Characterization tools}

The films were characterized by X-ray diffraction in $\theta-2 \theta$ scanning mode and by rocking curve mode on a D500 MOXTEK, using a $\mathrm{Cu} K_{\alpha}$ radiation $(\lambda=1.540598 \AA)$. The film cross-section observation performed by scanning electron microscopy (SEM) using a JEOL-type JSM $6400 \mathrm{~F}$ at an accelerating voltage of $5 \mathrm{kV}$. The structural study of films was also carried out by high resolution transmission electron microscopy (HRTEM, Hitachi HF 2000), with an acceleration voltage of $200 \mathrm{kV}$. Photoluminescence (PL) measurements were collected on a Jobin-Yvon Fluorolog 3 spectrometer using a xenon lamp (500 W) with excitation wavelength $=300 \mathrm{~nm}$, at room temperature.

Film thickness and substrate curvature were measured with a stylus profilometer DEKTAK 3030. As most commonly used, the stress in our films has been calculated by the bend-bending method, where the radius of the coated substrate curvature is determined and used to calculate the residual stress. The internal stress in the deposited film is calculated from the change in the substrate curvature from $1 / R_{0}$ for the uncoated substrate to $1 / R_{e}$ after film deposition, with the following Stoney formula [13]:

$$
\sigma=\frac{\left(E_{s} e_{s}^{2}\right)}{6 e\left(1-\nu_{s}\right)}\left(\frac{1}{R_{e}}-\frac{1}{R_{0}}\right),
$$

where $R_{0}$ is the radius of curvature of the Si substrate, $R_{e}$ is the curvature radius after film deposition, $E_{s}$ and $\nu_{s}$ are, respectively, the Young modulus and Poisson ratio of the substrate, $e_{s}$ and $e$ are the thickness of the substrate and the film, respectively.

\section{Results and discussions}

\subsection{Structural and morphological characterization}

Figure 2a,b presents the XRD patterns of $400 \mathrm{~nm}$ $\mathrm{ZnO}$ film deposited on silicon substrate and AlN buffers (600 $\mathrm{nm}$ and $1400 \mathrm{~nm}$ thick). The X-ray diffraction
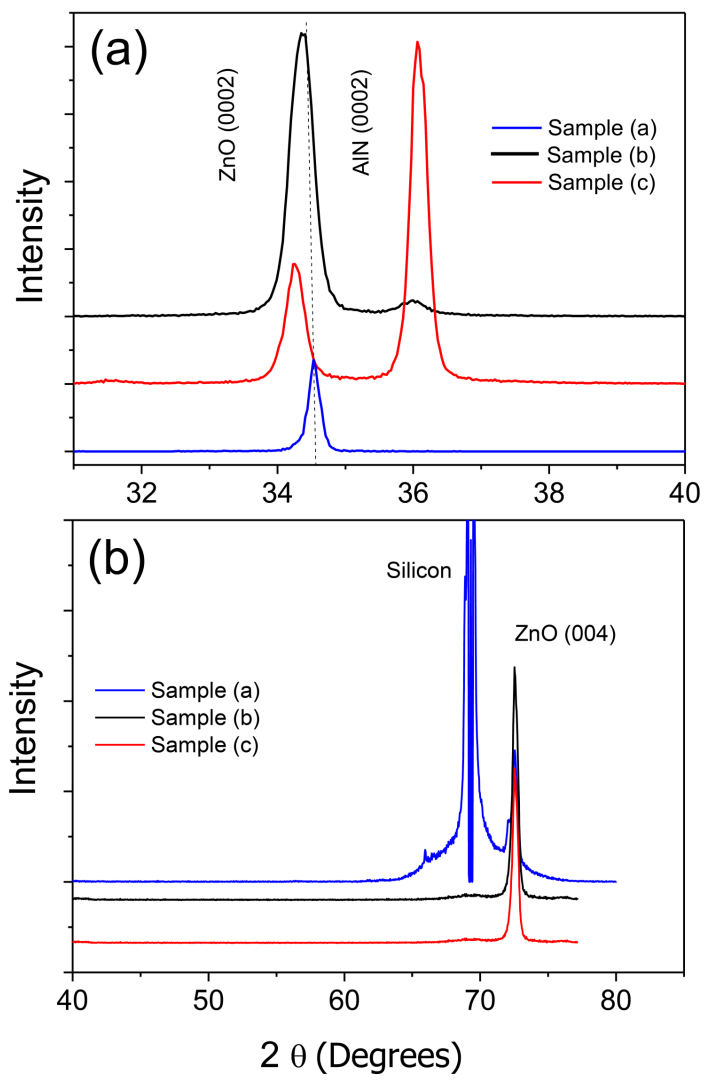

Fig. 2. XRD patterns of the $\mathrm{ZnO}$ films grown on silicon substrate and different AlN buffer layers (a) from 2030 $40^{\circ}$ and (b) extended interval from $40-80^{\circ}$. 


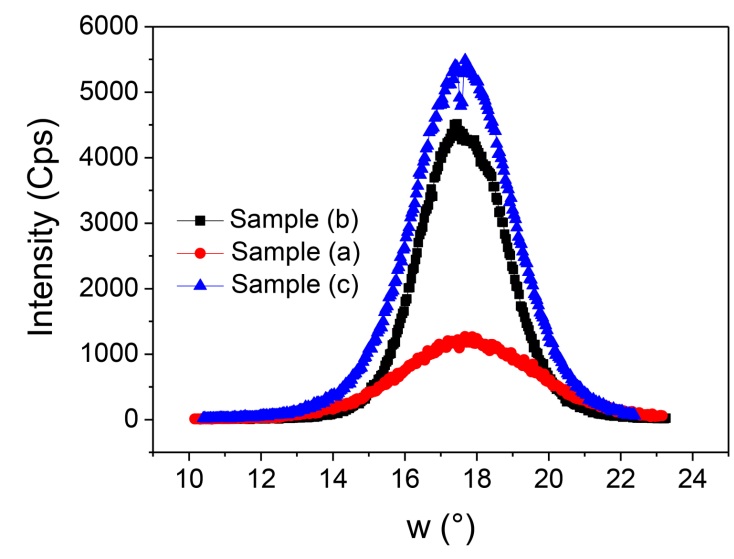

Fig. 3. The high-resolution XRD (0002) w-scan rocking curves of $\mathrm{ZnO}$ films grown on (a) silicon substrate, (b) $600 \mathrm{~nm}$ thick AlN buffer, and (c) $1400 \mathrm{~nm}$ thick AlN buffer.

(XRD) results reveal a remarkable crystallization of $\mathrm{ZnO}$ (0002) on AlN (0002) [1-3, 10]. Indeed, such result is confirmed by the FWHM of the (0002) $\mathrm{ZnO}$ in rocking curve (RC) mode (in Fig. 3), which is $4.3^{\circ}$ for $\mathrm{ZnO}$ on $\mathrm{Si}$ and $2.9^{\circ}$ and $2.4^{\circ}$ on $600 \mathrm{~nm}$ thick and $1400 \mathrm{~nm}$ thick AlN buffers, respectively. The extended XRD pattern (Fig. 2b) confirms the single orientation of $\mathrm{ZnO}$ deposited with and without the buffer layer. Moreover, one can observe an important shift of the $\mathrm{ZnO}$ (0002) diffraction peak from $34.53^{\circ}$ to $34.37^{\circ}$ and $34.25^{\circ}$, for samples a, b, and c, respectively. This indicates a change in the stress, which is compressive on Si and tensile on AlN. Such change in stress could have an impact on the optical properties of $\mathrm{ZnO}$ films. The stress calculated using Stoney's formula [13] was found to be $-0.7749 \mathrm{GPa}$ for $\mathrm{ZnO} / \mathrm{Si}$ (sample a) and $-0.6151 \mathrm{GPa}$ and $-0.4896 \mathrm{GPa}$ for sample $\mathrm{b}$ and $\mathrm{c}$, respectively. This implies that the stress of $\mathrm{ZnO}$ deposit is compressive on silicon, while the stress becomes tensile for $\mathrm{ZnO}$ deposited on AlN buffers.

Figure 4a, b shows the cross-section SEM images of $\mathrm{ZnO}$ film deposited on Si and $1400 \mathrm{~nm}$ thick AlN buffer layer, respectively. The $\mathrm{ZnO} / \mathrm{Si}$ film exhibits a dense and compact surface morphology compared to $\mathrm{ZnO}$ grown on AlN buffer. Indeed, the $\mathrm{ZnO}$ grown on $1400 \mathrm{~nm}$ thick AlN adopts a columnar structure, which in fact seems to be a continuous growth of the AlN buffer, which has already a columnar structure. The same structure of $\mathrm{ZnO}$ with columnar structure is also obtained for $600 \mathrm{~nm}$ thick AlN (not shown here). This is consistent with XRD results since both $\mathrm{ZnO}$ and $\mathrm{AlN}$ films have both a wurtzite structures with (0002) orientation.

In order to have an insight on the continuous growth of $\mathrm{ZnO}$ on AlN buffer, TEM was performed on $\mathrm{ZnO}$ deposited on $1400 \mathrm{~nm}$ thick AlN buffer. It should be noted that the same results were observed in the case of $\mathrm{ZnO}$ deposited on $600 \mathrm{~nm}$ thick AlN buffer (not shown here). The TEM micrograph of $\mathrm{ZnO}$ film deposited on the AlN buffer is displayed in Fig. 5a. In this figure, is it clear that

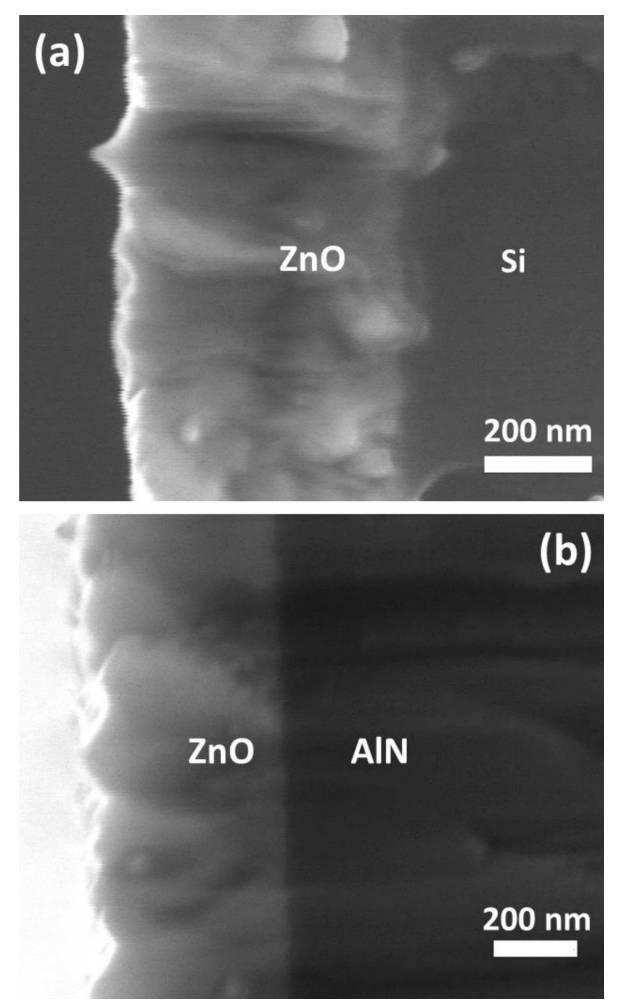

Fig. 4. Cross-section SEM images of $\mathrm{ZnO}$ deposited on (a) silicon and (b) $600 \mathrm{~nm}$ thick AlN buffer.

$\mathrm{ZnO}$ has a kind of continuous growth with well-aligned columns which are superposed on those of AlN buffer. The HRTEM image (Fig. 5b) of the rectangular part in Fig. 5a shows a kind of epitaxial growth of $\mathrm{ZnO}$ on AlN buffer with an interface that appears relatively abrupt. Such epitaxial growth is emphasized in Fig. 5c which is a high resolution of rectangular part in Fig. 5b. Indeed, the interface between $\mathrm{ZnO}$ film and AlN buffer is clearly abrupt, indicating a local epitaxial growth of $\mathrm{ZnO}$ on the AlN buffer. The electron diffraction patterns labelled 1, 2 and 3 on $\mathrm{AlN}$, the interface between $\mathrm{ZnO} / \mathrm{AlN}$ and $\mathrm{ZnO}$ film, respectively, confirm the single crystalline epitaxial nature of zinc oxide growth on AlN. In fact, well-aligned spots are observed in the SAED pattern of the interface $\mathrm{ZnO} / \mathrm{AlN}$ which are identical to those obtained for AlN or $\mathrm{ZnO}$ films. The sputtered $\mathrm{ZnO}$ film at the interface appears to be well crystallized and fully $c$-axis orientated. It is worth to notice that the ion bombardment had not destroyed the AlN crystalline structure during the beginning of $\mathrm{ZnO}$ growth thanks to AlN extreme resistance to amorphization during ion irradiation due to efficient dynamic defect annihilation [14].

\subsection{Photoluminescence measurements}

In order to evaluate the effect of AlN buffer layer on the PL properties of $\mathrm{ZnO}$, we first measured the PL properties of $\mathrm{ZnO}$ deposited directly on Si substrate without buffer. Figure 6 shows the PL spectra of $\mathrm{ZnO}$ thin film 

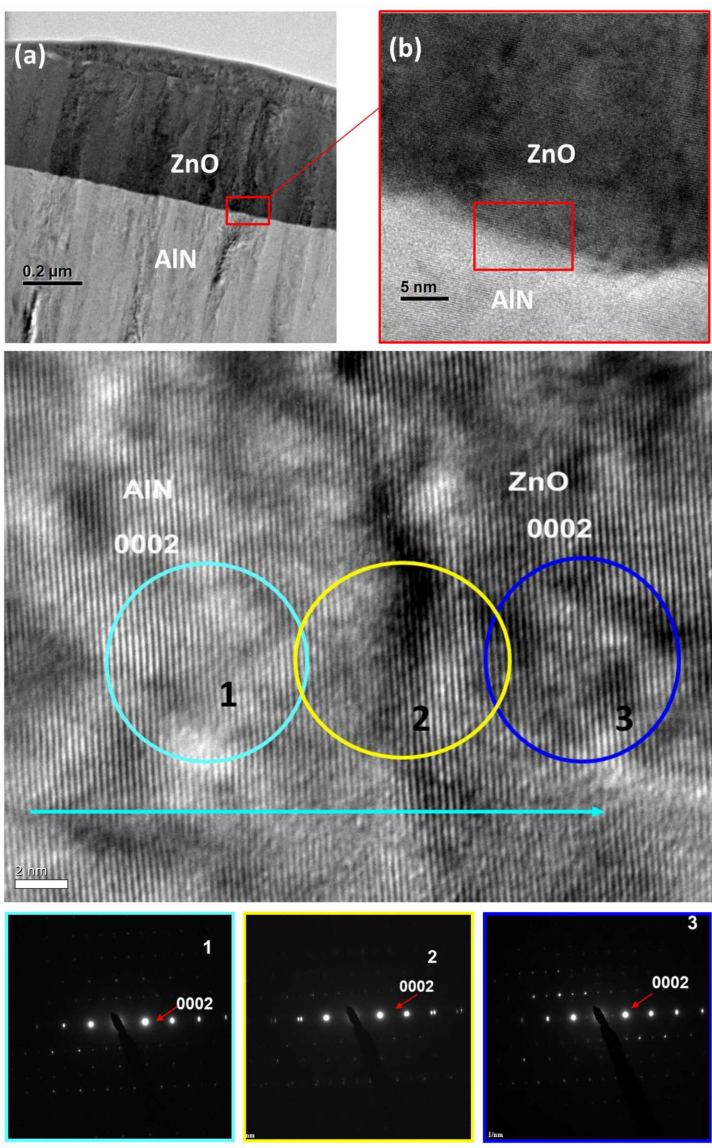

Fig. 5. TEM micrograph of $\mathrm{ZnO}$ film deposited on $1400 \mathrm{~nm}$ thick AlN buffer, (b) High resolution TEM image of the rectangular part of (a). The inset is the SAED of the rectangular part in (a). (c) High resolution TEM image of the rectangular part in (b).

grown on Si substrate. In Fig. 6, the strong peak at $389 \mathrm{~nm}(3.19 \mathrm{eV})$, corresponds to the band-edge emission of the $\mathrm{ZnO}$ due to recombination of excitons bound to donors $[15,16]$. The emission peaks at 411, 431, 500, $545 \mathrm{~nm}[17-19]$ can be ascribed to the intrinsic point defect, such as zinc vacancy, zinc interstitials, or oxygen vacancies [20]. However, these peaks may also originate from interference effects with the silicon layer. The spacing observed between them is about $40 \mathrm{~nm}$, which roughly corresponds to interference fringe spacing found for $1000 \mathrm{~nm}$ thick $\mathrm{ZnO}$ deposited on $\mathrm{Si}$ [21]. Indeed, these fringe spacings are due to the Fabry-Pérot oscillations that will be formed when a thin film is deposited on a smooth and refractive substrate. Multiple optical reflections occur due to differences in the refractive indices of the $\mathrm{ZnO}$ films, substrates, and the ambient (air in the present study) [21]. It must be noted that PL spectra free of fringe spacings have been reported in the case of $\mathrm{ZnO}$ films thinner than $70 \mathrm{~nm}$ or much thicker than $1.5 \mu \mathrm{m}$, which is not our case since our films have thickness of $400 \mathrm{~nm}$. In addition, when a thick buffer layer of AlN is used, these peaks are not observed as it will be shown hereafter.

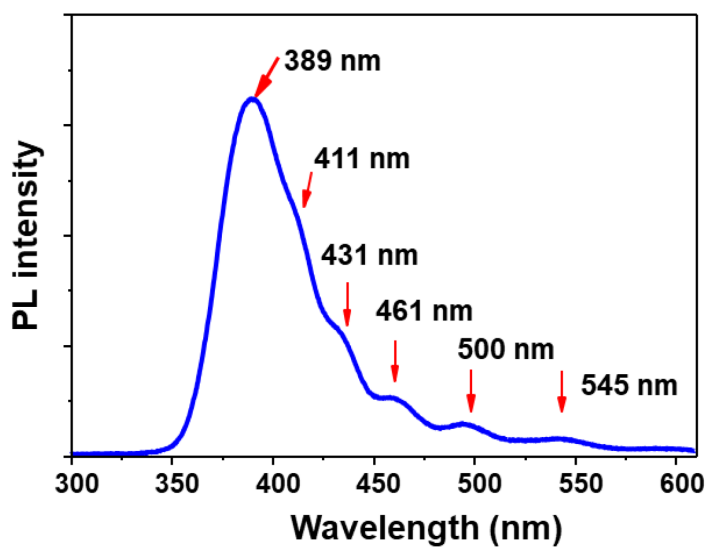

Fig. 6. Room temperature PL spectrum from $\mathrm{ZnO}$ thin film grown on Si substrate without a buffer layer.

It is also reported that the defect emissions depend on the fabrication process of $\mathrm{ZnO}[22]$. Transient PL spectra of $\mathrm{ZnO}$ film grown on Si substrate with different buffer layer (AlN) thicknesses of 600 and $1400 \mathrm{~nm}$ are shown in Fig. 7a,b. In Fig. 7a, a strong UV emission at $392 \mathrm{~nm}(3.16 \mathrm{eV})$ can be assigned to the near bandedge transition in $\mathrm{ZnO}$. The FX mode which is usually situated at $375.7 \mathrm{~nm}(3.3 \mathrm{eV})$ was not observed here [22] and therefore, any of its replicas FX-LO and FX-2LO, (band which is the free exciton (FX) and coupling of FX with orders of longitudinal optical (LO) phonon replicas) can be ruled out, even the FX-2LO is reported to be situated between 395 and $392 \mathrm{~nm}$ [23].

In addition, strong and weak emissions at 432 and $512 \mathrm{~nm}$, respectively, are observed in the case of $\mathrm{ZnO}$ deposited on $600 \mathrm{~nm}$ thick AlN buffer. Assuming that interference effects can be neglected here [21], these peaks may come from intrinsic point defects, such as zinc interstitials and oxygen vacancies [22-24], respectively. Concerning the emission, at $432 \mathrm{~nm}(2.8 \mathrm{eV})$ for $\mathrm{ZnO} / \mathrm{AlN}(600 \mathrm{~nm})$ it may originate from defect emission in $\mathrm{ZnO}[25,26]$ or synergistic effect between $\mathrm{ZnO}$ and $\mathrm{AlN}$ defects. In fact, a transition between the complexes of $\mathrm{Al}$ vacancies and oxygen on a nitrogen site in AlN and the $\mathrm{Zn}$ vacancies defect states in $\mathrm{ZnO}$ has been reported to be about $2.9 \mathrm{eV}$ [27], which is close to $2.8 \mathrm{eV}$ in our case.

The PL of $\mathrm{ZnO}$ deposited on thicker AlN buffer of $1400 \mathrm{~nm}$ manifest a strong and broad UV emission at $392.3 \mathrm{~nm}$ (equivalent to $3.16 \mathrm{eV}$ ) as shown in Fig. 7b, which indicates a decrease of the $\mathrm{ZnO}$ band gap [28]. One can notice from the PL spectra that the insertion of AlN buffer leads to red shift of UV emission by $4 \mathrm{~nm}$ and $4.3 \mathrm{~nm}$ for $600 \mathrm{~nm}$ and $1400 \mathrm{~nm}$ thick AlN buffers, respectively, suggesting a band gap decrease of $\mathrm{ZnO}$ when using AlN buffers. This can be attributed to stress effect in $\mathrm{ZnO}$ due to AlN buffer insertion [29]. Indeed, it has been demonstrated that the upward shift in the energy bandgap corresponds to the occurrence of compressive stress in the $\mathrm{ZnO}$ crystal [30], while a band-gap decrease is due to tensile stress [31]. This is consistent with the 

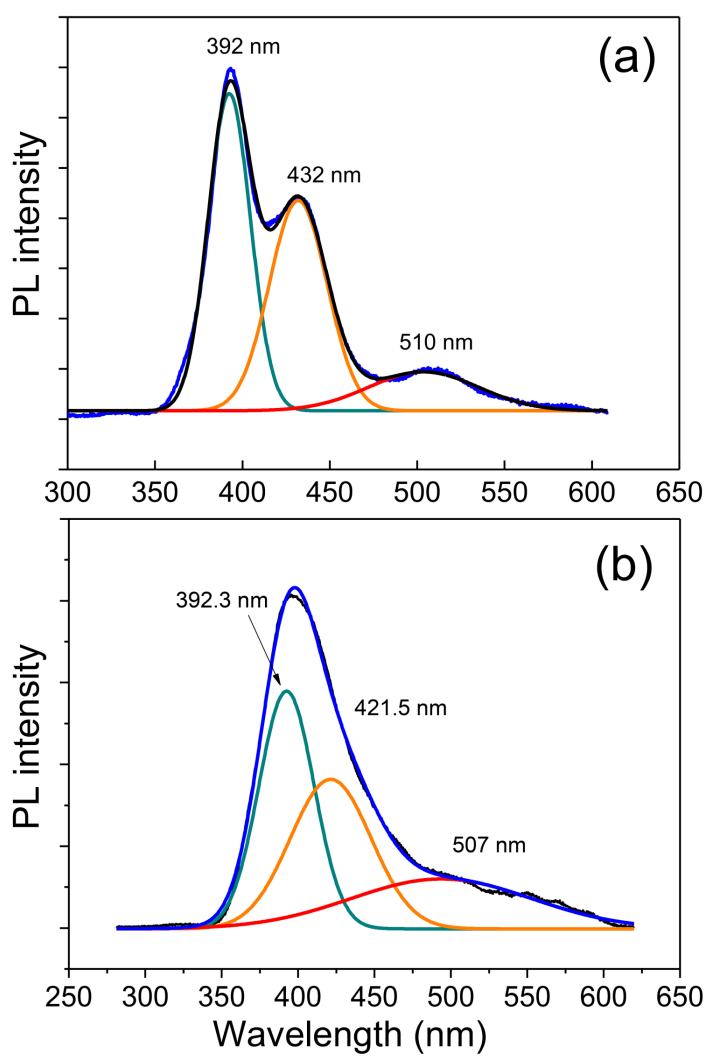

Fig. 7. Deconvoluted room temperature PL spectra from $\mathrm{ZnO}$ thin film grown on Si substrate with a buffer layer (a) $600 \mathrm{~nm}$, (b) $1400 \mathrm{~nm}$.

XRD results, where an increase in tensile stress is observed due to AlN buffer insertion. In addition, the defect emission (situated around $507 \mathrm{~nm}$ ) of the $\mathrm{ZnO}$ deposited on $1400 \mathrm{~nm}$ thick AlN is broader than the other peaks from the $\mathrm{ZnO} / \mathrm{Si}$ and $\mathrm{ZnO} / \mathrm{AlN}$ (600 nm) film, which is related to the defects that can originate from the void between columns [32]. It is worth to mention that the rocking curve measurements show only the orientation of $\mathrm{ZnO}$ in (0002) direction and do not give information about the other type of defects.

Finally, although, there is a local epitaxial growth of $\mathrm{ZnO}$ on $\mathrm{AlN}$ buffers, the defect emission that are originated from $\mathrm{ZnO}$, or $\mathrm{AlN}$ buffer or both were also present. In addition, no significant improvement of the band gap emissions were measured for films deposited on AlN buffers. Indeed, the local epitaxial growth was a kind of continuous local growth of the $\mathrm{ZnO}$ columns on those of AlN buffer as already shown in SEM/TEM images, thus, the $\mathrm{ZnO}$ deposited on AlN became less dense compared to $\mathrm{ZnO}$ deposited directly on $\mathrm{Si}$.

\section{Conclusions}

Epitaxial and $c$-axis oriented $\mathrm{ZnO}$ films were grown on $\mathrm{AlN} / \mathrm{Si}(100)$ substrate by RF magnetron sputtering at low temperature $\left(<100^{\circ} \mathrm{C}\right)$, which is an easy technique compatible with the current semiconductor technology. The PL results were somehow out of the traditional thought box, which supposes that epitaxial growth would enhance the band gap emission of $\mathrm{ZnO}$. The local epitaxial growth in our case was in the form of a continuous growth of $\mathrm{ZnO}$ columns on those of AlN, which led to less dense structure compared to that of $\mathrm{ZnO}$ grown directly on Si. This results to the appearance or increase of new defects such as zinc interstitials, thus, the PL signal was broadened and some defect emissions were enhanced. In addition, the defect from AIN buffer cannot be ruled out. Our results add a piece of information about the PL properties of $\mathrm{ZnO}$ with local epitaxial growth on AlN buffer.

\section{Acknowledgments}

This work was supported financially by School of Chemistry and Chemical Engineering, Shihezi University/Key Laboratory for Green Processing of Chemical Engineering of Xinjiang Bingtuan/Key Laboratory of Materials-Oriented Chemical Engineering of Xinjiang Uygur Autonomous Region/ Engineering Research Center of Materials-Oriented Chemical Engineering of Xinjiang Bingtuan, Beisi Road, Shihezi 832003, Xinjiang, People's Republic of China.

\section{References}

[1] F.E. Ghodsi, H. Absalan, Acta Phys. Pol. A 118, 659 (2010).

[2] D. Gültekin, M. Alaf, H. Akbulut, Acta Phys. Pol. A 123, 274 (2013).

[3] H. Eskalen, S. Ozan, U. Alver, S. Kerl, Acta Phys. Pol. A 127, 756 (2015).

[4] W. Li, Y-X. Xu, D. Wang, F. Chen, Z-K. Chen, Org. Electron. 57, 60 (2018).

[5] Y. Zeng, Y. Zhao, Y. Jiang, Surf. Interface Anal. 46, $602(2014)$.

[6] S.P. Wang, C.X. Shan, B. Yao, B.H. Li, J.Y. Zhang, D.X. Zhao, D.Z. Shen, X.W. Fan, Appl. Surf. Sci. 255, 4913 (2009).

[7] N.M. Sbrockey, S. Ganesan, III-Vs Review 17, 23 (2004).

[8] C. Jin, R. Narayan, A. Tiwari, H. Zhou, A. Kvit, J. Narayan, Mater. Sci. Eng. B 117, 348 (2005).

[9] A. Nahhas, H.K. Kim, J. Blachere, Appl. Phys. Lett. 78, 1511 (2001).

[10] H. Xiong, J.N. Dai, Xiong Hui, Y.Y. Fang, W. Tian, D.X. Fu, C.Q. Chen, Mingkai Li, Yunbin He, J. Alloys Compd. 554, 104 (2013).

[11] K. Kondo, M. Harada, N. Shibata, J. Ceram. Soc. Japan 110, 343 (2002).

[12] M. Fujita, N. Kawamoto, M. Sasajima, Y. Horikoshi, J. Vacuum Sci. Technol. B 22, 1484 (2004).

[13] J. Singh, S. Ranwa, J. Akhtar, M. Kumar, AIP Adv. 5, 067140 (2015).

[14] J.S. Williams, Mater. Sci. Eng. A 253, 8 (1998). 
[15] A. Sasaki, W. Hara, A. Matsuda, N. Tateda, S. Otaka, S. Akiba, K. Saito, T. Yodo, M. Yoshimoto, Appl. Phys. Lett. 86, 231911 (2005).

[16] V. Pawar, P.K. Jha, S.K. Panda, P.A. Jha, P. Singh, Phys. Rev. Appl. 9, 054001 (2018).

[17] B. Lin, Z. Fu, Y. Jia, Appl. Phys. Lett. 79, 943 (2001)

[18] Q.X. Zhao, P. Klason, M. Willander, H.M. Zhong, W. Lu, J.H. Yang, Appl. Phys. Lett. 87, 211912 (2005).

[19] N.O. Korsunska, L.V. Borkovska, B.M. Bulakh, L.Y. Khomenkova, V.I. Kushnirenko, I.V. Markevich, J. Lumin. 102, 733 (2003).

[20] A. Achour, M. Islam, S. Vizireanu, I. Ahmad, M.A. Akram, K. Saeed, G. Dinescu, J.-J. Pireaux, Nanomaterials 9, 794 (2019).

[21] Y.-G. Wang, N. Ohashi, H. Ryoken, H. Haneda, J. Appl. Phys. 100, 114917 (2006).

[22] Y. Gong, T. Andelman, G.F. Neumark, S. O'Brien, I.L. Kuskovsky, Nanoscale Res. Lett. 2, 297 (2007).

[23] U. Choppali, B.P. Gorman, Opt. Mater. 31, 143 (2008).
[24] M.A. Hashmi, K. Mahmood, A. Alib, M. Hasan, M. Raja, I. Hussain, M. Willander, ECS Trans. 35 149 (2011).

[25] M.K. Patra, K. Manzoor, M. Manoth, S.R. Vadera, N. Kumar, J. Lumin. 128, 267 (2008).

[26] D.H. Zhang, Z.Y. Xue, Q.P. Wang, J. Phys. D Appl. Phys. 35, 2837 (2002).

[27] J. Ding, H. Chen, H. Fu, Mater. Res. Bull. 95, 185 (2017).

[28] Munirah, Z.R. Khan, A. Aziz, M.S. Khan, M.U. Khandaker, Mater. Sci.-Poland 35, 243 (2017).

[29] V. Venkatachalapathy, A. Galeckas, I.-H. Lee, A.Yu. Kuznetsov, Physica B Condens. Matter 407, 1476 (2012)

[30] T. Prasada Rao, M.C. Santhosh Kumar, S. Anbumozhi Angayarkanni, M. Ashok, J. Alloys Compd. 485, 413 (2009).

[31] R.K. Sendi, S. Mahmud, J. Phys. Sci. 24, 1 (2013).

[32] M.S. Kim, K.G. Yim, J.-Y. Leem, J. Korean Phys. Soc. 59, 2354 (2011). 